\title{
1 How reproducible are surface areas calculated from the BET
}

\section{2 equation?}

Johannes W. M. Osterrieth ${ }^{a}$, James Rampersad ${ }^{a}$, David Madden $^{a}$, Nakul Rampal $^{a}$, Luka Skoric ${ }^{b}$, Bethany Connollya; Mark D. Allendorf , Vitalie Stavila ${ }^{c}$, Jonathan L. Sniderc; Rob Ameloot ${ }^{d}$, João Marreiros $^{d}$; Conchi Ania ${ }^{e}$; Diana Azevedo ${ }^{f}$, Enrique Vilarrasa-Garcia ${ }^{f}$ Bianca F. Santos ; Xian-He Bu $^{g}$, Ze Chang ${ }^{\prime}$; Hana Bunzen ${ }^{h}$; Neil R. Champness', Sarah L. Griffin'; Banglin Chen', Rui-Biao Lin'; Benoit Coasne ${ }^{k}$; Seth Cohen', Jessica C. Moreton'; Yamil J. Colon ${ }^{m}$; Linjiang Chen ${ }^{n}$, Rob Clowes ${ }^{n}$; François-Xavier Coudert'; Yong Cuip, Bang Hou ${ }^{p}$; Deanna M. D’Alessandro ${ }^{q}$, Patrick W. Doheny ${ }^{q}$; Mircea Dincăr', Chenyue Sun'; Christian Doonans, Michael Thomas Huxleys; Jack D. Evans'; Paolo Falcaro ${ }^{u}$, Raffaele Ricco ${ }^{u}$; Omar Farhav ${ }^{v}$, Karam B. Idrees ${ }^{v}$, Timur Islamogluv'; Pingyun Fengw , Huajun Yangw $^{w}$; Ross S. Forgan ${ }^{x}$, Dominic Bara ${ }^{x}$; Shuhei Furukaway, Eli Sanchez ${ }^{y}$; Jorge Gascon ${ }^{z}$, Selvedin Telalovic ; Sujit K. Ghosh ${ }^{a a}$, Soumya Mukherjee ${ }^{a a}$; Matthew R. Hill ${ }^{\text {ab }}$, Muhammed Munir Sadiq ${ }^{a b}$; Patricia Horcajada ${ }^{a c}$, Pablo Salcedo-Abraira ${ }^{a c}$; Katsumi Kaneko ${ }^{\text {ad }}$, Radovan Kukobat ${ }^{\text {ad }}$; Jeff Kenvin ${ }^{a e}$; Seda Keskin ${ }^{\text {af; Susumu Kitagawa }}{ }^{a g}$, Ken-ichi Otake ${ }^{a g}$; Ryan P. Lively ${ }^{\text {ah }}$, Stephen J. A. DeWitt ${ }^{\text {ah }}$ Phillip Llewellyn ; Bettina V. Lotsch ${ }^{a j, a k}$, Sebastian T. Emmerlingaj,ak, Alexander M. Pütz ${ }^{a j, a k}$; Carlos Martí-Gastaldo ${ }^{a l}$, Natalia Padialal; Javier García-Martínez ${ }^{a m}$, Noemi Linares $^{a m}$; Daniel Maspoch ${ }^{\text {an,ao, }}$ Jose A. Suárez del Pino ao; Peyman Moghadam ${ }^{a p}$, Rama Oktavian ${ }^{a p}$; Russel E. Morris ${ }^{a q}$, Paul S. Wheatley ${ }^{\text {aq; }}$ Jorge Navarro ${ }^{\text {ar; }}$ Camille Petit ${ }^{a s}$, David Danacias; Matthew J. Rosseinsky ${ }^{\text {at }}$, Alexandros P. Katsoulidisat; Martin Schröder ${ }^{a u}$, Xue Han ${ }^{a u}$, Sihai Yang ${ }^{a u}$; Christian Serre ${ }^{a v}$, Georges Mouchaham $^{a v}$; David S. Shollah, Raghuram Thyagarajan ${ }^{\text {ah }}$ Daniel Siderius $^{\psi, \varphi, a w} ;$ Randall Q. Snurr $^{a x}$, Rebecca B. Goncalves ${ }^{a y}$; Shane Telfer ${ }^{a z}$, Seok J. Lee ${ }^{a z}$; Valeska P. Ting ${ }^{\text {ba }}$, Jemma L. Rowlandson ${ }^{\text {ba }}$; Takashi Uemura ${ }^{b b}$, Tomoya liyuka ${ }^{b b}$; Monique A. van der Veen ${ }^{b c}$, Davide Rega ${ }^{b c}$; Veronique Van Speybroeck ${ }^{b d}$, Sven M. J. Rogge ${ }^{b d}$, Aran Lamaire ${ }^{b d}$; Krista S. Walton ${ }^{\text {ah }}$, Lukas W. Bingelah; Stefan Wuttke ${ }^{b e, b f}$, Jacopo Andreo ${ }^{b e, b f}$; Omar Yaghibg,bh, Bing Zhang ${ }^{\text {bg }}$; Cafer T. Yavuz ${ }^{b i}$, Thien S. Nguyen ${ }^{b i}$; Felix Zamora ${ }^{b j}$, Carmen Montoro ${ }^{b j}$; Hongcai Zhou ${ }^{b k}$, Angelo Kirchon ${ }^{b k}$; and David Fairen-Jimenez ${ }^{a,{ }^{*}}$

a The Adsorption \& Advanced Materials Laboratory ( $\left.A^{2} M L\right)$, Department of Chemical Engineering \& Biotechnology, University of Cambridge, Philippa Fawcett Drive, Cambridge CB3 OAS, UK

${ }^{b}$ Cavendish Laboratory, University of Cambridge, JJ Thomson Avenue, CB3 OHE, Cambridge, United Kingdom

c Sandia National Laboratories, 7011 East Avenue, Livermore, California 94550, United States ${ }^{d}$ cMACS, Department of Microbial and Molecular Systems (M²S), KU Leuven, 3001 Leuven, Belgium e CEMHTI, CNRS (UPR 3079), Université d'Orléans, 45071 Orléans, France

${ }^{\dagger}$ LPACO2/GPSA, Department of Chemical Engineering, Federal University of Ceará, 60455-760 Fortaleza (CE), Brazil g School of Materials Science and Engineering, National Institute for Advanced Materials, Nankai University, Tianjin 300350, China

$37{ }^{\mathrm{h}}$ Chair of Solid State and Materials Chemistry, Institute of Physics, University of Augsburg,

38 Universitaetsstrasse 1, Augsburg 86159, Germany

39 ISchool of Chemistry, University of Nottingham, University Park, Nottingham, NG7 2RD UK 
jDepartment of Chemistry, University of Texas at San Antonio, One UTSA Circle, San Antonio, TX 782490698, USA

kUniv. Grenoble Alpes, CNRS, LIPhy, 38000 Grenoble, France

'Department of Chemistry and Biochemistry, University of California, San Diego, La Jolla, California, 92093 USA

m Department of Chemical and Biomolecular Engineering, University of Notre Dame, Notre Dame, IN, 46556, USA

${ }^{n}$ Leverhulme Research Centre for Functional Materials Design, Materials Innovation Factory and Department of Chemistry, University of Liverpool, Liverpool, UK - Chimie ParisTech, PSL University, CNRS, Institut de Recherche de Chimie Paris, 75005 Paris, France p School of Chemistry and Chemical Engineering, Shanghai Jiaotong University, 800 Dongchuan Road, Minhang District, Shanghai a School of Chemistry, The University of Sydney, New South Wales, 2006, Australia r Department of Chemistry, Massachusetts Institute of Technology, Cambridge, Massachusetts 02139, USA ${ }^{\mathrm{s}}$ Centre for Advanced Nanomaterials and Department of Chemistry, The University of Adelaide, North Terrace, Adelaide, SA 5000, Australia

t Department of Inorganic Chemistry, Technische Universität Dresden, Bergstrasse 66, 01062, Dresden, Germany

u Institute of Physical and Theoretical Chemistry, Graz University of Technology, Graz, Austria

v Department of Chemistry and International Institute of Nanotechnology, Northwestern University, 2145 Sheridan Road, Evanston, Illinois 60208, United States w Department of Chemistry, University of California, Riverside, California 92521, USA

${ }^{x}$ WestCHEM School of Chemistry, University of Glasgow, Glasgow, UK

y Institute for Integrated Cell-Material Sciences, Kyoto University, Yoshida, Sakyo-ku, Kyoto 606-8501, Japan ${ }^{z}$ KAUST Catalysis Center (KCC), King Abdullah University of Science and Technology, P.O.Box 4700, 23955-6900, Thuwal-Jeddah, Kingdom od Saudi Arabia

aa Department of Chemistry, Indian Institute of Science Education and Research (IISER), Pune, Dr. Homi Bhabha Road, Pashan, Pune 411008, India ab CSIRO, Private Bag 33, Clayton South MDC, VIC 3169, Australia and Department of Chemical Engineering, Monash University, Clayton, VIC 3168, Australia

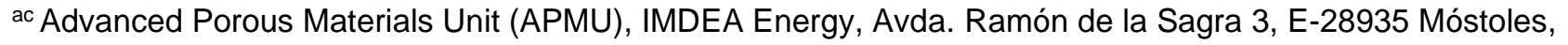
Madrid, Spain

${ }^{\text {ad }}$ Research Initiative for Supra-Materials, Shinshu University, Nagano, Japan

ae Micromeritics Instrument Corporation, Norcross, GA 30093, USA

af Department of Chemical and Biological Engineering, Koc University, Rumelifeneri Yolu 34450 Sariyer, Istanbul, Turkey

ag Institute for Integrated Cell-Material Sciences (WPI-iCeMS), Kyoto University Institute for Advanced Study (KUIAS), Kyoto University, Yoshida Ushinomiya-cho, Sakyo-ku, Kyoto 606-8501, Japan an School of Chemical \& Biomolecular Engineering, Georgia Institute of Technology, Atlanta, GA 30332, USA ai CNRS / Aix-Marseille Univ. / TOTAL

aj Max Planck Institute for Solid State Research, Heisenbergstrasse 1, 70569 Stuttgart, Germany ak Department of Chemistry, University of Munich (LMU), Butenandtstrasse 5-13, 81377 Munich, Germany al Instituto de Ciencia Molecular (ICMol), Universitat de València, Paterna 46980, València, Spain am Laboratorio de Nanotecnología Molecular, Departamento de Química Inorgánica, Universidad de Alicante, Ctra. San Vicente-Alicante s/n, E-03690 San Vicente del Raspeig, Spain 
an ICREA, Pg. Lluís Companys 23, Barcelona, 08010, Spain

ao Catalan Institute of Nanoscience and Nanotechnology (ICN2), CSIC and the Barcelona Institute of Science and Technology. Campus UAB, Bellaterra, 08193 Barcelona, Spain

ap Department of Chemical and Biological Engineering, The University of Sheffield, United Kingdom

aq School of Chemistry, University of St Andrews, North Haugh, St Andrews, KY16 9ST, UK

ar Departamento de Química Inorgánica, Universidad de Granada, 18071 Granada, Spain

as Barrer Centre, Department of Chemical Engineering, Imperial College London, London, U.K., SW7 2AZ

at Materials Innovation Factory, Department of Chemistry, University of Liverpool, Liverpool, L7 3NY, UK

au School of Chemsitry, The University of Manchester, Manchester, U.K. M13 9PL

av Institut des Matériaux Poreux de Paris, Ecole Normale Supérieure, ESPCI Paris, CNRS, PSL University, 75005 Paris, France

aw Chemical Sciences Division, National Institute of Standards and Technology, Gaithersburg, Maryland USA 20899-8320

ax Departments of Chemical \& Biological Engineering, Northwestern University, 2145 Sheridan Road, Evanston, Illinois 60208, USA

ay Department of Chemistry, Northwestern University, 2145 Sheridan Road, Evanston, Illinois 60208, USA

az MacDiarmid Institute for Advanced Materials and Nanotechnology, Institute of Fundamental Sciences, Massey University, Palmerston North, 4442 New Zealand ba Department of Mechanical Engineering, University of Bristol, Bristol BS8 1TR, U.K.

bb Department of Advanced Materials Science, Graduate School of Frontier Sciences, The University of Tokyo, 5-1-5 Kashiwanoha, Kashiwa, Chiba 277-8561, Japan

bc Department of Chemical Engineering, Delft University of Technology, van der Maasweg 9, 2629HZ Delft, the Netherlands

bd Center for Molecular Modeling (CMM), Ghent University, Technologiepark 46, B-9052 Zwijnaarde, Belgium be BCMaterials, Basque Center for Materials, Applications and Nanostructures, UPV/EHU Science Park, 48940, Leioa, Spain

bf IKERBASQUE, Basque Foundation for Science, 48009, Bilbao, Spain

bg Department of Chemistry, University of California-Berkeley; Kavli Energy Nanoscience Institute at UC Berkeley

bh Berkeley Global Science Institute, Berkeley, California 94720, United States

bi Department of Chemical and Biomolecular Engineering, Korea Advanced Institute of Science and Technology (KAIST), Yuseong-gu, 34141 Daejeon, Korea

bj Departamento de Química Inorgánica, Universidad Autónoma de Madrid, 28049 Madrid, Spain

bk Chemistry Department -Texas A\&M University

$\psi$ Official contribution of the National Institute of Standards and Technology (NIST), not subject to copyright in the United States of America

$\varphi$ Certain commercially available items may be identified in this paper. This identification does not imply recommendation by NIST, nor does it imply that it is the best available for the purposes described

*E-mail: df334@cam.ac.uk 
124 Porosity and surface area analysis play a prominent role in modern materials science, where 125 their determination spans the fields of natural sciences, engineering, geology and medical 126 research. At the heart of this sits the Brunauer-Emmett-Teller (BET) theory, ${ }^{[1]}$ which has been 127 a remarkably successful contribution to the field of materials science. The BET method was 128 developed in the 1930s for open surfaces but is now the most widely used metric for the estimation of surface areas of micro- and mesoporous materials. ${ }^{[2]}$ Since the BET method was first developed, there has been an explosion in the field of nanoporous materials with 131 the discovery of synthetic zeolites, ${ }^{[3]}$ nanostructured silicas, ${ }^{[4-6]}$ metal-organic frameworks 132 (MOFs), ${ }^{[7]}$ and others. Despite its widespread use, the manual calculation of BET surface 133 areas causes a significant spread in reported areas, resulting in reproducibility problems in 134 both academia and industry. To prove this, we have brought together 60 labs with strong 135 track records on the study of nanoporous materials. We provided eighteen already measured raw adsorption isotherms and asked these researchers to calculate the corresponding BET areas. This round-robin exercise resulted in a wide range of values for each isotherm. We demonstrate here that the reproducibility of BET area determination from identical isotherms is a largely ignored issue, raising critical concerns over the reliability of reported BET areas in micro- and mesoporous materials in the literature. To solve this major issue, we have developed a new computational approach to accurately and systematically determine the BET area of nanoporous materials. Our software, called BET Surface Identification (BETSI), expands on the well-known Rouquerol criteria and makes, for the first time, an unambiguous BET area assignment possible.

The Brunauer-Emmett-Teller (BET) equation is arguably one of the most used equations in physical chemistry and porosimetry. Since its conception in the $1930 \mathrm{~s}^{[1]}$ to estimate open surfaces whilst working with non-microporous adsorbents of the time such as $\mathrm{Fe} / \mathrm{Cu}$ catalysts, silica gel and charcoal, it found widespread use in the characterisation of synthetic zeolites. ${ }^{[3]}$ Furthermore, it has gained considerable momentum following the discovery of more complex porous materials such as mesoporous silicas ${ }^{[4-6]}$, porous coordination polymers $(\mathrm{PCPs})^{[8]}$, metal-organic frameworks (MOFs) ${ }^{[7]}$ and covalent organic frameworks (COFs) ${ }^{[9]}$. Novel porous materials are of significant academic and industrial interest due to their applications in gas storage and separation, ${ }^{[10-13]}$ catalysis, ${ }^{[14]}$ sensing, ${ }^{[15,16]}$ and drug delivery. ${ }^{[17]}$ To assess their adsorptive properties, Langmuir was the first to relate gas adsorption isotherms to surface areas, assuming only monolayer adsorption. ${ }^{[18]}$ This was in contrast to Dubinin's proposition of micropore volumes for microporous materials. ${ }^{[19]}$ Langmuir's adsorption theory was later extended to multilayer adsorption, resulting in the titular BET model.

157 Even though the BET theory was not developed for describing adsorption in the microporosity, the 158 BET area is now the de facto standard for the characterisation of any porous material. Indeed, it has 159 been recognized by the International Union of Pure and Applied Chemistry (IUPAC) as "the most 160 widely used procedure for evaluating the surface area of porous and finely-divided materials". $[2,20]$ 161 Furthermore, it has been an International Organization for Standardization (ISO) standard for surface 
area determination since $1995 .{ }^{[21]}$ This makes it, arguably, the most important figure of merit for porous materials, including microporous ones. Looking at the literature, it is clear that the idea of monolayer coverage or even the concept of surface area are necessarily idealised and therefore could be inaccurate descriptions for microporous materials. ${ }^{[22]}$ Indeed, IUPAC warns users to apply "extreme caution [when using the BET equation] in the presence of micropores. (...) [The BET area] represents an apparent surface area, which may be regarded as a useful adsorbent 'fingerprint'."[2] This more nuanced understanding of the BET area is mirrored in the writing of Rouquerol et al., "the meaning of the BET surface is (...) that it embraces the major part of the amount of adsorptive in energetic interaction with the surface." ${ }^{[22]}$ Despite these cautionary words, the BET area remains a deeply engrained metric in the fields of physical chemistry and materials science. Given the broad use of the BET equation, it is not surprising to see that much has been written on the applicability and the accuracy of the BET theory - that is, its model of the adsorption process - and on the reproducibility of the raw data, i.e. the adsorption isotherm. ${ }^{[23-26]}$

Since the development of the first porous materials, there has been a sharp rise in the design of highly ordered and structured porous materials (Fig. S1). ${ }^{[27,28]}$ The advent of materials with more complex pore networks and dynamic frameworks through material design strategies such as reticular chemistry has given rise to reported BET areas in excess of $8,000 \mathrm{~m}^{2} \mathrm{~g}^{-1} \cdot{ }^{[11,29-34]}$ Often, these modern materials have complex adsorption isotherms which are more problematic or ambiguous to fit to the BET model, e.g. several steps can occur due to different pore types and/or flexibility being present in the material. ${ }^{[35]}$ In response, a new generation of porosimetry equipment with pressure transducers capable of recording high-resolution gas adsorption isotherms at ultra-low pressures $\left(<10^{-5} \mathrm{mmHg}\right)$ has been developed. However, reliance on manual calculations of surface areas using the BET method remains commonplace. In this context, 'manual' refers to the judicious selection of a pressure range by a scientist, be it through a self-developed spreadsheet or commercial software. This raises the question of the reproducibility of BET calculations from the same isotherm. An adsorption isotherm with 150 points has more than 10,000 consecutive combinations of points, all of which are potentially correct fitting ranges and will return different BET areas. The answer to the question of which is the optimal fitting region is far from obvious, and the consequence of any irreproducibility or different interpretations are serious. Consider two groups synthesizing the same compound and reporting two different BET areas; Sample A is reported to have a BET area of 1,500 $\mathrm{m}^{2} \mathrm{~g}^{-1}$ and Sample B's reported BET area is $2,000 \mathrm{~m}^{2} \mathrm{~g}^{-1}$. Unless there is a common standard and protocol for calculating BET areas, we cannot say for certain that the quality and adsorption performance of Sample A is lower than that of Sample B. Indeed, the lack of reproducibility of MOF syntheses and adsorption performance, by comparing reported BET areas, has been highlighted already, but the natural spread of BET calculations was not included in the analysis. ${ }^{[36]}$

The eponymously named Rouquerol criteria (Section S2, Supplementary Information) aim to ensure good practice in identifying a valid fitting range, and, as such, they have found widespread acceptance in the literature and have been adopted in both IUPAC and ISO standards. ${ }^{[2,20-22,24,25,37]}$ 
Despite this safeguard, we herein propose that current BET area calculations are irreproducible for two reasons: first, the Rouquerol criteria are indeterminate in identifying the correct fitting region, as they apply to multiple regions simultaneously. Second, even if they were determinate, they are too cumbersome and lengthy to implement and are therefore often neglected in practice. This dilemma is reminiscent of the Skeptic's Argument from Gorgias, here paraphrased: i) the BET area does not exist (e.g., for microporous materials); ii) even if it exists, it cannot be systematically and unequivocally calculated (i.e., determined by the Rouquerol criteria).

To prove our hypothesis and to assess the current spread of BET calculation results, we have shared a dataset of 18 isotherms (reported as relative pressure vs. amount adsorbed), already measured, and representing four classes of micro- and mesoporous materials (zeolites, mesoporous silicas, MOFs and COFs) with 60 laboratories with expertise in adsorption science and synthesis of porous materials. In this round-robin exercise, we asked the researchers to calculate the BET areas in the way they saw most fit. More details about the specific materials and the adsorption isotherms, sampled both from our laboratory and from the NIST/ARPA-E database, ${ }^{[38]}$ are included in the 214 Supplementary Information, Section S10. To avoid any recognition bias, all isotherms were anonymised and scaled-off arbitrarily. Figure 1a shows the large spread of results obtained from manual calculations of BET areas in the round-robin experiment, the full details can be found anonymised in the Supplementary Information, tabulated in Section S3, and represented graphically in Section S4. Most groups (90\%) reported using the Rouquerol criteria in their manual calculation, $23 \%$ used a commercial software package, and $6 \%$ used a self-developed code. Details on the methods for each group can be found in Section S11 of the Supporting Information. Bar a few exceptions, virtually no two groups of experts reported identical BET areas for any given isotherm. We observed a spread of at least $300 \mathrm{~m}^{2} \mathrm{~g}^{-1}$ for each isotherm; however, that number was significantly higher for some individual isotherms. For NU-1104, a modern MOF with substantial porosity (isotherm Figure 1b), ${ }^{[32]}$ the highest estimate of $9,341 \mathrm{~m}^{2} \mathrm{~g}^{-1}$ and the lowest estimate of $1,757 \mathrm{~m}^{2} \mathrm{~g}^{-1}$ differed by an astonishing $7,584 \mathrm{~m}^{2} \mathrm{~g}^{-1}$, making the highest estimate more than five times higher than the lowest estimate. The spread of values for frequently reproduced MOFs such as HKUST-1, MOF-5 and ZIF-8 was slightly smaller than that of literature cited values. ${ }^{[36]}$ While this observation affirms the natural assumption that material synthesis and isotherm measurement play a more important role in determining the BET area than the calculation, we can nevertheless attribute a significant portion of this spread to current BET fittings. The results of this social study demonstrate that there is significant variation in BET area calculations from the same isotherm, as it is extremely unlikely for two researchers to select identical fitting regions. At this point, we propose a novel method that not only systematically selects an optimal fitting region but does so by eliminating all other hypothetical fitting regions. With thousands of consecutive combinations of points, the large number of potential fittings is impossible to carry out manually. 
a
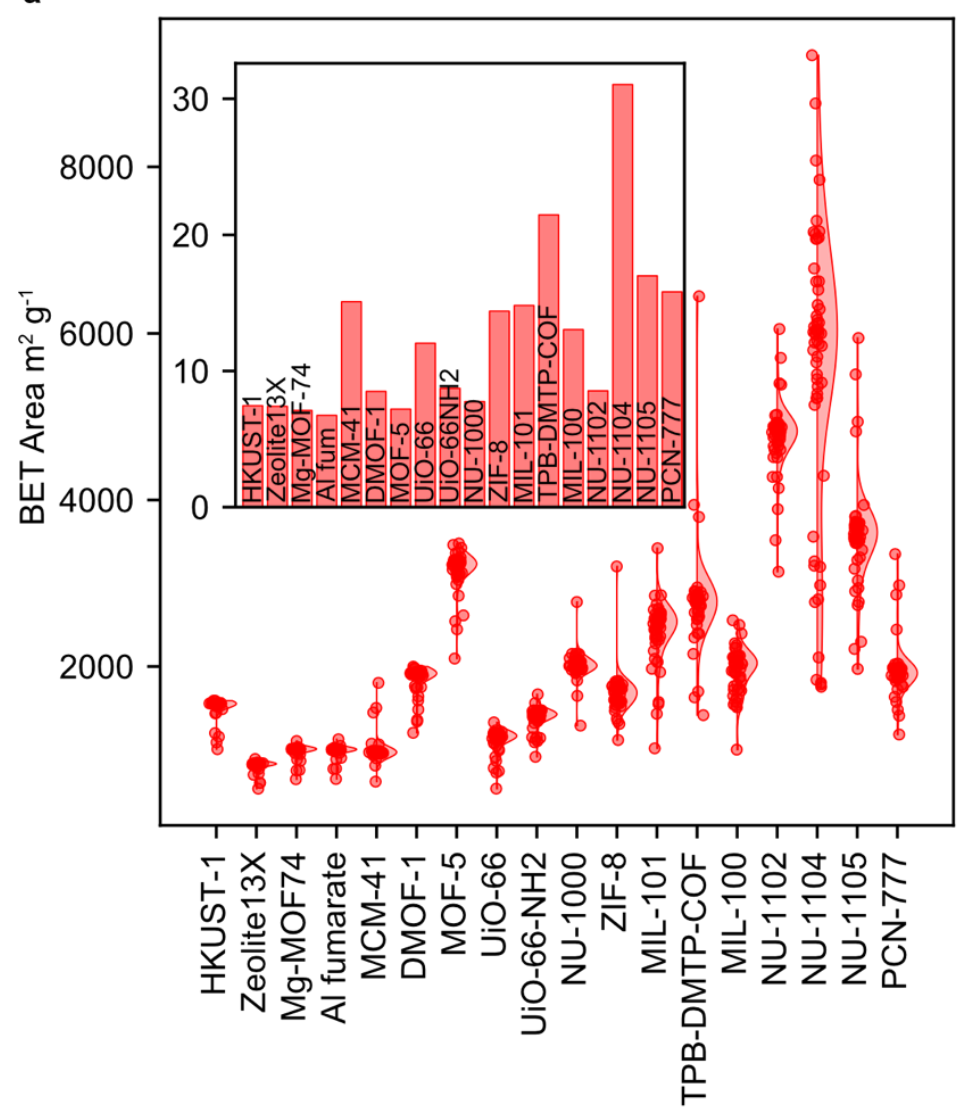

b

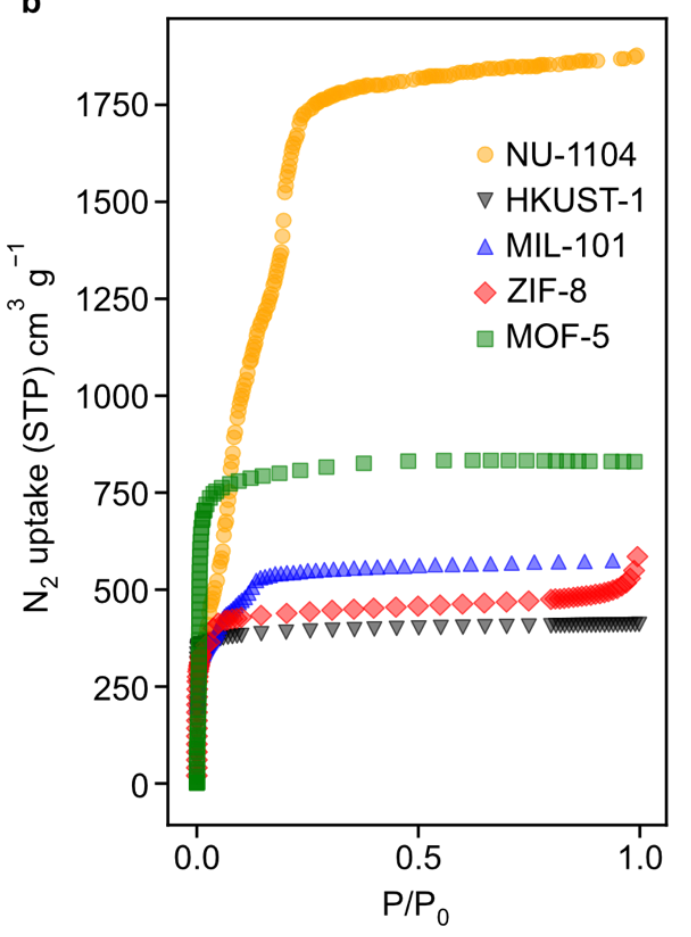

236

Figure 1 | Round-robin results of BET area calculation. a, Distribution of BET areas from identical isotherms as calculated by 60 laboratories with an expertise in adsorption science and synthesis of porous materials. Superimposed are normalised probability distribution functions obtained by kernel density estimation. Inset shows the coefficient of variation (relative standard deviation) of results for each material $\mathbf{b}$, Exemplary isotherms for materials shown in a. The large spread of BET areas reported for NU-1104 is due to the unusual shape of its adsorption isotherm, making manual BET fits difficult.

To solve the problem of manual BET fitting, we developed a computational tool for BET analysis, BET Surface Identification (BETSI). This tool makes an unambiguous calculation of the BET area based on the original Rouquerol criteria but modified to prevent manual interaction, requiring only the adsorption isotherm as input data. As such, the results obtained from the round-robin evaluation were compared with the BETSI calculations to assess the inter-rater reliability of manual BET calculations. Figure 2 shows the working principle of the BETSI algorithm on a simplified $\mathrm{N}_{2}$ adsorption isotherm at $77 \mathrm{~K}$ for ZIF-8 (full details can be found in the Supporting Information, Section S5). First, the linearized BET equation is fitted to a particular region of the isotherm using an ordinary least-squares (OLS) regression (Figure 2a). The top panel shows the isotherm with a fitting region highlighted in red, and the OLS regression is shown below. The plot insets show the checks against the Rouquerol criteria (Figure $\mathbf{2 b}$ ). If all criteria are met, the fitting is passed. This calculation is looped over all data intervals of at least 10 points on the isotherm. The resulting BET fits are stored in a large $n \times n$ matrix, where the (j,i)-matrix element corresponds to a fitting region starting at the $j^{\text {th }}$ point and ending on the $\mathrm{i}^{\text {th }}$-point (Figure 2c). All valid fitting results are output and plotted against the percentage error under the $4^{\text {th }}$ Rouquerol criterion (Figure 2d). Alongside, BETSI outputs all 
other BET parameters, such as monolayer capacity and the C constant, as well as full regression diagnostics (Section S5).

a

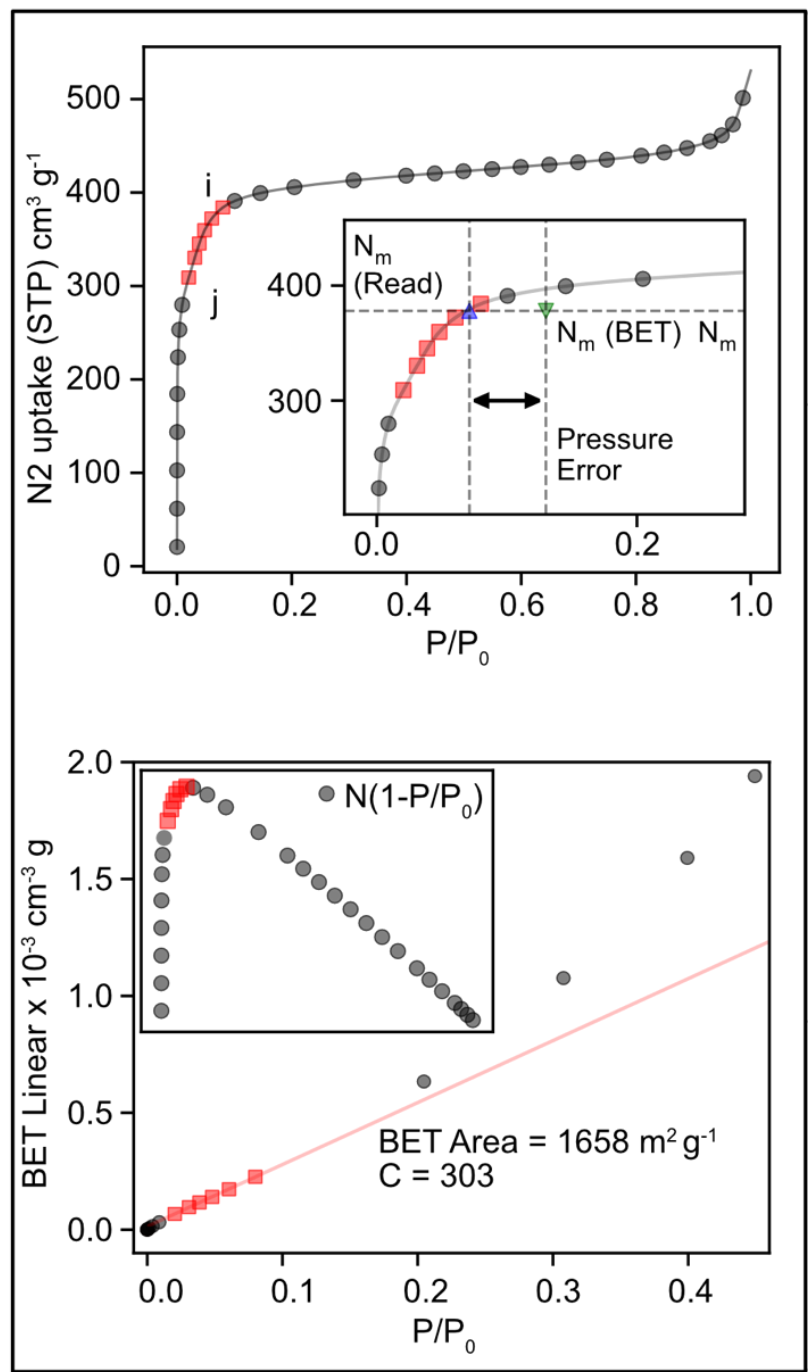

b Rouquerol Criteria

1. $N\left(1-P / P_{0}\right)$ vs $P$ must be monotonically increasing

2. The $\mathrm{C}$ constant must be positive

3. The pressure corresponding to the monolayer loading, when reported on the isotherm, $\mathrm{N}_{\mathrm{m}}$ (Read), must lie in the fitting range

4. $\quad \mathrm{N}_{m}$ (Read) and $\mathrm{N}_{m}(\mathrm{BET})$, the monolayer loading calculated from BET must not differ by more c than $20 \%$.

Rouquerol Filter Consistent Inconsistent

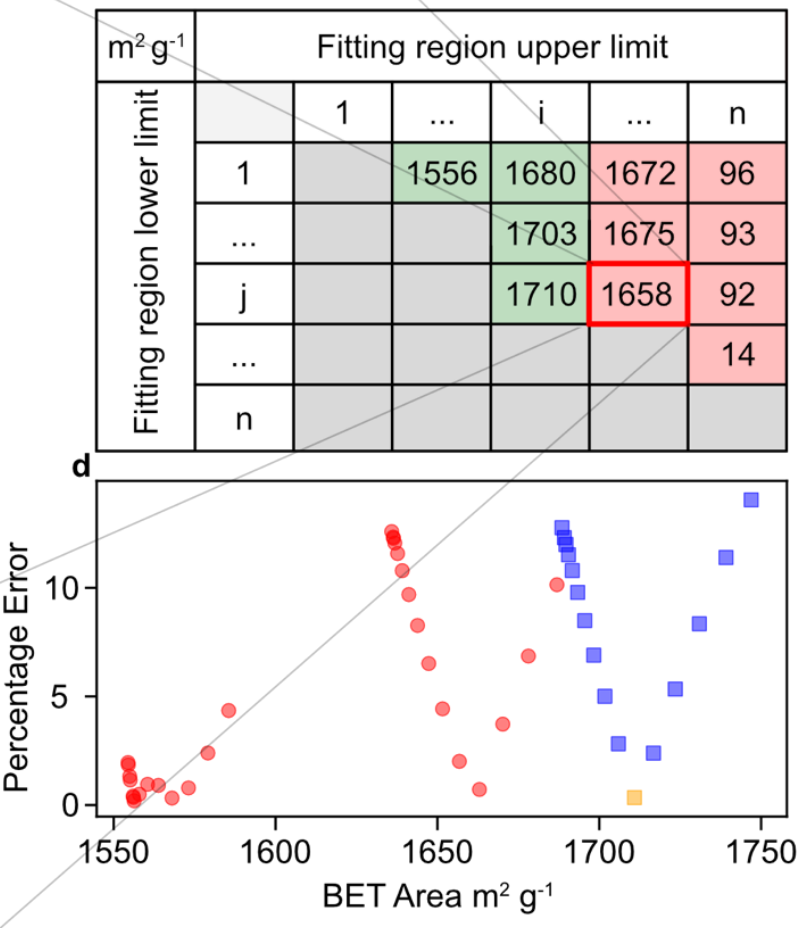

Figure 2 | Working principle for BETSI algorithm. a, The isotherm is shown with a particular fitting region highlighted in red. The linear BET equation is applied, and an ordinary least squares regression is applied to the fitting region. b, Subsequent checks against the Rouquerol criteria ${ }^{[24]}$ are performed (insets) and $\mathbf{c}$, valid fits are passed, The analysis shown in a is repeated for all consecutive combinations of points on the isotherm. A results matrix with $\mathrm{n} \times \mathrm{n}$ dimensionality stores all acceptable and rejected fits $\mathbf{d}$, All acceptable BET areas are output and plotted against the percentage error under the $4^{\text {th }}$ Rouquerol criterion. (a, top inset). All BET areas ending on the highest permissible point under the $1^{\text {st }}$ Rouquerol criterion (a, bottom inset, maximum in $\mathrm{N}\left(1-\mathrm{P} / \mathrm{P}_{0}\right.$ function) are labelled as the isotherm knee and shown in blue. The BETSI Optimal BET area (yellow) belongs to the isotherm knee group and has the lowest percentage error under the $4^{\text {th }}$ Rouquerol criterion.

Since multiple fittings comply with the Rouquerol criteria (Figure 2c-d), BETSI demonstrates that an unambiguous assignment of the BET area is impossible under the Rouquerol criteria alone. This proves our hypothesis that the criteria in their current form are indeterminate. For the prototypical ZIF-8 isotherm, a flexible MOF with narrow windows, ${ }^{\left[{ }^{[5]}\right.}$ valid BET areas fall within a range of 1,550 and $1,750 \mathrm{~m}^{2} \mathrm{~g}^{-1}$ (Figure 2c-d). BETSI assigns special relevance to fitting ranges that end on the highest permissible point, which are usually dictated by the $1^{\text {st }}$ Rouquerol criterion, and labels these as the isotherm knee. Beyond the isotherm knee, adsorptive activity decreases 
277 rapidly as the pores are mostly filled and the internal surfaces are saturated. Within this subset of

278 BET areas, the BETSI optimum is chosen as the one with the smallest percentage error under the $2794^{\text {th }}$ Rouquerol criterion, thus making the BET assignment unambiguous.

280 Next, we ran BETSI on the isotherms distributed in the round-robin experiment. In all cases, the 281 spread of potential BETSI results (i.e., those in agreement with the Rouquerol criteria) was 282 considerably narrower than that obtained by manual calculation (Table 1). Figure $3 a$ shows the 283 individual results from the social experiment and the comparison with the BETSI results; the 284 corresponding variation coefficients are shown in Section S6 and an alternative representation 285 normalised to the BETSI range is shown in Section S7. Since most groups reported using the 286 Rouquerol criteria to calculate their BET areas, this substantiates our second hypothesis - that the 287 manual implementation of the Rouquerol criteria is cumbersome and difficult to carry out in practice. 288 For instance, in the case of NU-1104, the range of estimates decreases from $7,500 \mathrm{~m}^{2} \mathrm{~g}^{-1}$ in the 289 social study to $235 \mathrm{~m}^{2} \mathrm{~g}^{-1}$ under BETSI. Interestingly, some isotherms gave much larger spreads of 290 results than others, suggesting that the BET model does not describe them as naturally and thus 291 they are more susceptible to problems associated with the Rouquerol criteria. Unsurprisingly, we 292 also observed this trend in the round-robin evaluation. To further investigate the goodness of the 293 isotherm fittings, we define the BETSI variation coefficient as the relative standard deviation of BETSI 294 results, and the pass rate as the number of BET fits that pass under the Rouquerol criteria as a 295 fraction of all potential fits. Further, the Hit Rate expresses the fractional number of BET areas 296 calculated in the round-robin exercise that lie within the BETSI range. Figure 3b demonstrates the 297 correlation between the pass rate, the BETSI variation coefficient, and the Hit Rate. Simply put, the 298 more BET fits are valid, the greater the spread of possible BET areas is, and the more likely 299 researchers are to satisfy the Rouquerol criteria in manual calculations. To account for the non-equal 300 spacing of points on all different isotherms, the pressure-adjusted pass rate expresses the total sum 301 of pressure intervals that fit Rouquerol criteria as a fraction of the sum of all pressure intervals of the 302 hypothetical fitting ranges (Section S8). From Figure 3b, we classify adsorption isotherms into three 303 broad categories, types A, B and C (Figure 3c). While it is difficult to generalise about the shape of 304 these isotherms, we still offer some discussion of common features. Type A isotherms fit the BET 305 model 'best'. Under BETSI, they have a relatively high pass rate and return a fairly narrow spread of 306 results. Examples include materials such as Al-fumarate, NU-1000, Zeolite-13X and MCM-41. Many 307 of these isotherms do not have strongly pronounced isotherm knees and some have mesoporous 308 steps. Hit rates greater than $70 \%$ are generally observed for these materials, suggesting that the 309 majority of researchers did not struggle with the fittings. Type B isotherms only fit the BET model 310 over a very limited range. These have extremely low pass rates, meaning that only few BET fits are 311 valid, which in turn will be spread narrowly. Examples include MOF-5, DMOF-1, NU-1104, HKUST312 1, and NU-1105. For the latter, out of 9,409 hypothetical 10-point fits, only one is permissible under 313 the Rouquerol criteria. Such prohibitively low pass rates make the correct BET assignment by hand 314 virtually impossible and demonstrate the need for computational support. In contrast to type A 
315 isotherms, type B isotherms often have sharp isotherm knees following strong adsorptive 316 interactions at low relative pressures. Isotherms with more complex shapes such as NU-1104 also 317 appear in this category. Type $\mathrm{C}$ isotherm fittings are arguably the most problematic. They have high 318 pass rates and, concomitantly, they return large spreads of BET results. Typical materials that fit into 319 this category are MIL-101, MIL-100, TPB-DMTP-COF and PCN-777. Like type A isotherms, these 320 have rounded isotherm knees, which appear at higher relative pressures. It is for these materials 321 that the necessity to extend the Rouquerol criteria is demonstrated and the BETSI algorithm makes 322 an unambiguous BET assignment possible.

323 Table 1 | Results of BETSI analysis and round-robin evaluation for the isotherms used in the study. 324 Material, isotherm of material under investigation; BETSI, optimal BET area predicted by BETSI; BETSI 325 Range, full spread of BET areas that pass under BETSI; BETSI Variation Coefficient, relative standard 326 deviation of BET areas that pass under BETSI; Pass Rate, number of BET areas that pass under BETSI expressed as a fraction of all hypothetical fittings; Round-robin Average, mean of BET areas calculated in round-robin evaluation; Round-robin Range, full spread of BET areas determined in round-robin evaluation; Round-robin Variation Coefficient, relative standard deviation of BET areas calculated in round-robin evaluation; Hit Rate, fraction of BET areas calculated in the round-robin evaluation that lie within the BETSI

\begin{tabular}{ccccccccc}
\hline Material & $\begin{array}{c}\text { BETSI } \\
\mathbf{m}^{2} \mathbf{g}^{-1}\end{array}$ & $\begin{array}{c}\text { BETSI } \\
\text { Range } \\
\mathbf{m}^{2} \mathbf{g}^{-1}\end{array}$ & $\begin{array}{c}\text { BETSI } \\
\text { Variation } \\
\text { Coefficient } \\
\%\end{array}$ & $\begin{array}{c}\text { Pass } \\
\text { Rate } \\
\%\end{array}$ & $\begin{array}{c}\text { Round- } \\
\text { robin } \\
\text { Average } \\
\mathbf{m}^{2} \mathbf{g}^{-1}\end{array}$ & $\begin{array}{c}\text { Round- } \\
\text { robin } \\
\text { Range } \\
\mathbf{m}^{\mathbf{2}} \mathbf{g}^{-1}\end{array}$ & $\begin{array}{c}\text { Round- } \\
\text { robin } \\
\text { Variation } \\
\begin{array}{c}\text { Coefficient } \\
\%\end{array}\end{array}$ & $\begin{array}{c}\text { Hit } \\
\text { Rate } \\
\%\end{array}$ \\
\hline HKUST-1 & 1556 & 8 & 0.090 & 2.419 & 1520 & 583 & 7.451 & 52 \\
Zeolite13X & 833 & 4 & 0.140 & 0.538 & 813 & 356 & 7.405 & 35 \\
Mg-MOF74 & 1010 & 5 & 0.114 & 2.300 & 990 & 459 & 7.101 & 48 \\
Al-Fumarate & 1007 & 14 & 0.398 & 1.736 & 989 & 478 & 6.740 & 60 \\
MCM-41 & 1001 & 60 & 1.573 & 3.329 & 994 & 1186 & 15.090 & 85 \\
DMOF-1 & 1924 & 4 & 0.074 & 0.107 & 1860 & 795 & 8.500 & 15 \\
MOF-5 & 3255 & 20 & 0.250 & 0.071 & 3170 & 1382 & 7.203 & 13 \\
UiO-66 & 1145 & 91 & 1.901 & 0.870 & 1120 & 796 & 12.045 & 65 \\
UiO-66-NH 2 & 1424 & 285 & 4.710 & 1.722 & 1388 & 750 & 8.727 & 48 \\
NU-1000 & 2068 & 160 & 1.619 & 4.218 & 2014 & 1486 & 7.752 & 80 \\
ZIF-8 & 1709 & 188 & 3.718 & 0.861 & 1672 & 2085 & 14.396 & 58 \\
MIL-101 & 2446 & 680 & 8.353 & 3.738 & 2429 & 2404 & 14.816 & 78 \\
TPB-DMTP- & & & & & & & & \\
COF & 2875 & 711 & 7.298 & 5.375 & 2787 & 5031 & 21.472 & 80 \\
MIL-100 & 2199 & 616 & 7.611 & 12.111 & 1964 & 1554 & 13.042 & 78 \\
NU-1102 & 4931 & 204 & 1.139 & 0.862 & 4770 & 2915 & 8.541 & 38 \\
NU-1104 & 5684 & 235 & 1.327 & 0.024 & 5553 & 7584 & 31.047 & 5 \\
NU-1105 & 3635 & 0 & 0.000 & 0.011 & 3585 & 3974 & 16.991 & 0 \\
PCN-777 & 2079 & 483 & 5.624 & 6.960 & 1946 & 2168 & 15.814 & 87 \\
\hline
\end{tabular}




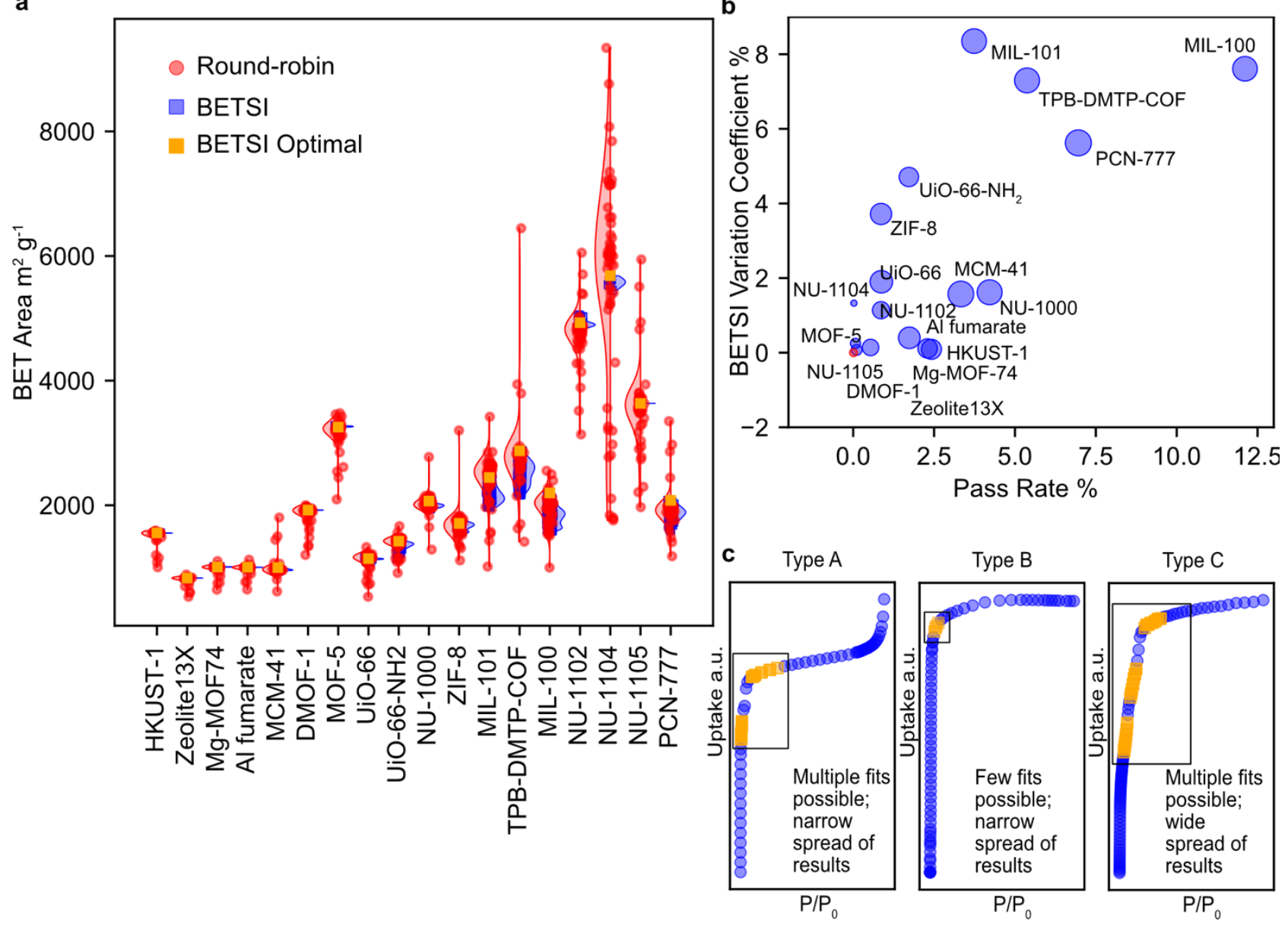

Figure 3 | Social study results vs BETSI results. a, Distribution of BET areas for identical isotherms from the social study (red) and BETSI (blue). Superimposed is the BETSI optimum (yellow). Note that the distributions of values obtained by BETSI are considerably narrower in all cases than those in the social study b. Plot of the BETSI Variation Coefficient (relative standard deviation of BETSI results) against the pass rate (fraction of valid fits against all hypothetical ones). Bubble size scales with the hit rate, the fraction of results from the social study that lie within the BETSI range. Red symbols have a hit rate of zero. Note the positive correlation between all three parameters $\mathbf{c}$, Isotherm fit classifications. Type A fits have a relatively wide fitting window, within which multiple fits are possible, but return a relatively narrow spread of BET results. Type B fits have a narrow fitting window and concomitantly return a narrow set of spread of results. Type $C$ fits have wide fitting windows, which translates to multiple passable fits and a wide spread of permissible BET areas.

\section{Outlook}

BET theory is a great success story. Developed in the 1930s for non-microporous, open surfaces, it continues to this day to be applied to modern adsorbents with complex porosity. Despite the advances from classical density functional theory (DFT) methods, the BET area will likely continue playing a crucial role in porosimetry for decades to come, with impacts in energy research, transport, medical applications and climate-change mitigation. In light of these future developments, it will become increasingly important to share critical scientific metrics reliably to find a common language to report both academic and industrial progress.

Here, we have demonstrated the difficulties in unambiguously determining BET areas from adsorption isotherms, which in turn affect the assessment of material quality and reproducibility. These problems arise from imperfect and insufficient manual calculations and can only be met using 
modern computational methods. Furthermore, we propose BETSI as a step towards greater transparency and criticality in determining BET areas. We stress here that it is neither the function nor the purpose of BETSI to eliminate doubt and treat a particular BET area as 'true'. Researchers should remain aware of the limitations of BET theory when applied to microporous adsorbents in general and when BET areas are reported, the pressure range and number of points used should always be stated. We further recommend here that isotherms must be reported transparently and in detail, i.e. semi-log representation to show the low-pressure regions. The 'experiment' is the adsorption isotherm - not the BET area.

\section{Online Content}

Source and extended data, details of author contributions and detailed instructions about the use of BETSI are included in the supplementary information.

Isotherm data reported with this paper are included in the NIST/ARPA-E Database of Novel and Emerging Adsorbent Materials, https://adsorption.nist.gov, and may be accessed directly at https://adsorption.nist.gov/isodb/index.php?DOI=10.XXXX/YYYYY\#biblio.

\section{References}

[1] S. Brunauer, P. H. Emmett, E. Teller, J. Am. Chem. Soc. 1938, 60, 309-319.

[2] M. Thommes, K. Kaneko, A. V. Neimark, J. P. Olivier, F. Rodriguez-Reinoso, J. Rouquerol, K. S. W. Sing, Pure Appl. Chem. 2015, 87, 1051-1069.

[3] R. Cid, R. Arriagada, F. Orellana, J. Catal. 1983, 80, 228-230.

[4] D. Zhao, J. Feng, Q. Huo, N. Melosh, G. H. Fredrickson, B. F. Chmelka, G. D. Stucky, Science 1998, 279, 548-552.

[5] J. S. Beck, J. C. Vartuli, W. J. Roth, M. E. Leonowicz, C. T. Kresge, K. D. Schmitt, C. T. W. Chu, D. H. Olson, E. W. Sheppard, S. B. McCullen, et al., J. Am. Chem. Soc. 1992, 114, 10834-10843.

[6] A. Corma, Chem. Rev. 1997, 97, 2373-2419.

[7] H. C. Zhou, J. R. Long, O. M. Yaghi, Chem. Rev. 2012, 112, 673-674.

[8] S. Kitagawa, R. Kitaura, S. I. Noro, Angew. Chemie - Int. Ed. 2004, 43, 2334-2375.

[9] C. S. Diercks, O. M. Yaghi, Science 2017, 355, DOI 10.1126/science.aal1585.

[10] J. Li, J. Sculley, H. Zhou, Chem. Rev. 2012, 112, 869-932.

[11] O. K. Farha, A. Ö. Yazaydin, I. Eryazici, C. D. Malliakas, B. G. Hauser, M. G. Kanatzidis, S. T. Nguyen, R. Q. Snurr, J. T. Hupp, Nat. Chem. 2010, 2, 944-948.

[12] B. Li, H.-M. Wen, W. Zhou, B. Chen, J. Phys. Chem. Lett. 2014, 5, 3468-3479.

[13] P. Z. Moghadam, T. Islamoglu, S. Goswami, J. Exley, M. Fantham, C. F. Kaminski, R. Q. Snurr, O. K. Farha, D. Fairen-Jimenez, Nat. Commun. 2018, 9, 1378-1385.

[14] A. Corma, H. García, F. X. Llabrés i Xamena, Chem. Rev. 2010, 110, 4606-4655.

[15] L. E. Kreno, K. Leong, O. K. Farha, M. Allendorf, R. P. Van Duyne, J. T. Hupp, Chem. Rev. 2012, $112,1105-1125$. 
[16] W. P. Lustig, S. Mukherjee, N. D. Rudd, A. V. Desai, J. Li, S. K. Ghosh, Chem. Soc. Rev. 2017, 46, 3242-3285.

[17] P. Horcajada, R. Gref, T. Baati, P. K. Allan, G. Maurin, P. Couvreur, G. Férey, R. E. Morris, C. Serre, Chem. Rev. 2012, 112, 1232-1268.

[18] I. Langmuir, J. Am. Chem. Soc. 1918, 40, 1361-1403.

[19] Y. K. Tovbin, Russ. Chem. Bull. 1998, 47, 637-643.

[20] K. S. W. Sing, D. H. Everett, R. A. W. Haul, L. Moscou, R. A. Pierotti, J. Rouquerol, T. Siemieniewska, Pure Appl. Chem. 1985, DOI 10.1351/pac198557040603.

[21] ISO [International Organization for Standardization], Ref. number ISO 2010, DOI 10.1007/s11367011-0297-3.

403

[22] J. Rouquerol, F. Rouquerol, P. Llewellyn, G. Maurin, K. S. W. Sing, Adsorption by Powders and Porous Solids: Principles, Methodology and Applications: Second Edition, 2013.

405

406

[23] F. Ambroz, T. J. Macdonald, V. Martis, I. P. Parkin, Small Methods 2018, 2, 1800173.

407

408

[24] D. A. Gómez-Gualdrón, P. Z. Moghadam, J. T. Hupp, O. K. Farha, R. Q. Snurr, J. Am. Chem. Soc. 2016, 138, 215-24.

[25] K. S. Walton, R. Q. Snurr, J. Am. Chem. Soc. 2007, 129, 8552-8556.

[26] J. Park, J. D. Howe, D. S. Sholl, Chem. Mater. 2017, 29, 10487-10495.

[27] P. Z. Moghadam, A. Li, S. B. Wiggin, A. Tao, A. G. P. Maloney, P. A. Wood, S. C. Ward, D. Fairen-

[28] M. E. Davis, Nature 2002, 417, 813-821.

[29] H. Furukawa, K. E. Cordova, M. O'Keeffe, O. M. Yaghi, Science 2013, 341, 1230444-1230444.

[30] O. M. Yaghi, J. Am. Chem. Soc. 2016, 138, 15507-15509.

O. K. Farha, I. Eryazici, N. C. Jeong, B. G. Hauser, C. E. Wilmer, A. A. Sarjeant, R. Q. Snurr, S. T. Nguyen, A. Ö. Yazaydin, J. T. Hupp, J. Am. Chem. Soc. 2012, 134, 15016-15021.

[32] T. C. Wang, W. Bury, D. A. Gómez-Gualdrón, N. A. Vermeulen, J. E. Mondloch, P. Deria, K. Zhang, P. Z. Moghadam, A. A. Sarjeant, R. Q. Snurr, et al., J. Am. Chem. Soc. 2015, 137, 3585-3591.

[33] Z. Chen, P. Li, R. Anderson, X. Wang, X. Zhang, L. Robison, L. R. Redfern, S. Moribe, T. Islamoglu, D. A. Gómez-Gualdrón, et al., Science 2020, 368, 297-303.

[34] I. M. Hönicke, I. Senkovska, V. Bon, I. A. Baburin, N. Bönisch, S. Raschke, J. D. Evans, S. Kaskel, Angew. Chemie - Int. Ed. 2018, 57, 13780-13783.

[35] D. Fairen-Jimenez, S. A. Moggach, M. T. Wharmby, P. A. Wright, S. Parsons, T. Düren, J. Am. Chem. Soc. 2011, 133, 8900-8902.

[36] M. Agrawal, R. Han, D. Herath, D. S. Sholl, Proc. Natl. Acad. Sci. U. S. A. 2020, 117, 877-882.

[37] J. Rouquerol, P. Llewellyn, F. Rouquerol, Stud. Surf. Sci. Catal. 2007, 160, 49-56.

[38] D.W. Siderius, V.K. Shen, R.D. Johnson III, and R.d. van Zee, Eds., NIST/ARPA-E Database of Novel and Emerging Adsorbent Materials, National Institute of Standards and Technology, 


\section{Round-robin evaluation}

$\mathrm{N}_{2}$ adsorption isotherms of 18 different materials (Supplementary Information Section S10) were sent to international collaborators: HKUST-1, ZIF-8, NU-1000, MIL-101, UiO-66, Al fumarate, Zeolite13X, Mg-MOF-74, UiO-66-NH 2 , MOF-5, DMOF-1, MCM-41, TPB-DMTP-COF, MIL-100, NU1102, NU-1104, NU-1105, and PCN-777; they were anonymised and labelled A-R respectively. Note that this is not the order in which the isotherms appear in the paper. The isotherms were sampled from our own group measurements and from the NIST Adsorption Database. Arbitrary scaling factors were introduced to minimise recollection bias of the isotherms. The isotherms were sent out in .csv format. All colleagues received the same email with the same set of instructions (Section S9): To calculate the BET area from the data in the way they saw most fit and to report a rough estimate of how long it took them to calculate them. An anonymised one-page summary of each lab's own account of their calculation can be found in the Supporting Information, Section S11.

For easier data handling, once rescaled, all results were rounded to the next integer. None of the data points has been eliminated. The data is presented as a jitter plot for each material, with a superimposed kernel-density estimation obtained in python.

\section{BETSI}

The BETSI algorithm, including executables, is fully published in the Electronic Supplementary Information (https://github.com/fairen-group/betsi-gui). The programme is written in python and uses principally the numpy library. Looped linear regressions over all consecutive combinations of at least three points, perform full BET analyses and store the fitting parameters in $n \times n$ results matrices, where the (j,i)-matrix element denotes a linear regression from the $\mathrm{j}$ 'th to the i'th point on the isotherm. Binary pass/fail matrices with the same dimensionality are used independently to assess compliance with linearity and fitting criteria. The 'filtering' of BET areas is achieved by element-wise matrix multiplication of the results matrices and the pass/fail matrices. This allows independent 'activation' and 'deactivation' of the criteria and observing the effects on the results. The minimum fitting requirement of ten points is coded in a pass/fail matrix to allow for some minimum point flexibility, as is the cut-off value for $R^{2}$ of 0.995 . To avoid low-leverage non-linearity in the linear region, the first Rouquerol criterion has been extended to also require the linearised BET function to increase monotonically with $\mathrm{P} / \mathrm{P}_{0}$, as well as $\mathrm{N}\left(1-\mathrm{P}_{\mathrm{P}}\right)$. The third and fourth Rouquerol criteria are implemented through a 10,000 point Pchip interpolation of the isotherm to reconstruct the $\mathrm{N}_{\mathrm{m}}$ (Read). As the third and fourth criteria require the $N_{m}(B E T)$ to be a real value, i.e. they require $C$ to be positive, the second criterion cannot be independently deactivated from the third and the fourth. The associated logic has been written into the programme. Following the BETSI filtering by multiplication of results and pass/fail matrices, the isotherm knee is identified as the subset of BET areas whose fitting region end on the highest permissible pressure point. In most cases this will be the highest permissible point under the first Rouquerol criterion. The optimal BETSI prediction is chosen as the 
471 fitting region with the lowest percentage error under the fourth criterion and belonging to the isotherm

472 knee subset.

473 BETSI only requires the adsorption isotherm as input data and returns six plots used to validate 474 the results: the isotherm itself, with the optimal linear region highlighted as well as the BET fit; the 475 'Rouquerol representation' of the isotherm, $N\left(1-P / P_{0}\right)$ plotted against $P / P_{0}$; the linearised plot with 476 the OLS regression and the regression parameters; the filtered percentage error vs BET areas plot 477 with the isotherm knee and optimal BET area highlighted; the filtered monolayer-loadings plot 478 showing all permissible monolayer loadings on the isotherm; and the statistical distribution of 479 permissible BET areas with a boxplot. Additionally, BETSI returns four regression diagnostics plots 480 which can be used to assess whether the assumptions of OLS regression have been met: The 481 Residuals vs Fitted values plot can be used to visually inspect whether the residuals are normally 482 distributed around the regression line, and similar information can be obtained from the QQ-plot. 483 Finally, the Scale-Location plot can be used to assess whether the distribution of studentized 484 residuals is homoscedatic or heteroscedatic and the Residuals vs Leverage plot can be used to 485 identify high-leverage points that have an abnormally large influence on the regression line.

\section{Comparison between round-robin evaluation and BETSI results}

487 Statistical analysis of the results was performed in python. The BETSI variation coefficient and the 488 Round-robin variation coefficient are standard deviations relative to the average of each set. The 489 pass rate for each isotherm is the number of permissible BET fits as a fraction of all consecutive 490 combination of points. To account for non-equal spacing of the points on each isotherm, the 491 pressure-adjusted pass-rate is obtained by integrating along the pressure axis and dividing the total 492 sum of permissive pressure intervals by the sum of all consecutive pressure intervals. The hit rate is 493 the fractional number of BET areas calculated in the round-robin evaluation that lie within the BETSI 494 range.

\section{Author Contributions}

496 J.W.M.O. and D.F-J. designed the study. J.W.M.O. and D.M. collected and curated the dataset of 497 isotherms shared among coauthors. J.W.M.O., J. R., N. R., L. S. and B. C. developed BETSI code. 498 All coauthors calculated the BET areas from the pre-measured isotherms. J.W.M.O., D. M and D.F499 J. co-wrote the paper. All authors discussed the results and contributed to the editing of the 500 manuscript.

\section{Acknowledgements}

502 This project has received funding from the European Research Council (ERC) under the European 503 Union's Horizon 2020 research and innovation programme (NanoMOFdeli), ERC-2016-COG 504 726380, Innovate UK (104384) and EPSRC IAA (IAA/RG85685).

505 O.K.F. and R.Q.S. acknowledge funding from the U.S. Department of Energy (DE-FG02506 08ER15967). 
R.S.F. and D.B. acknowledge funding from the European Research Council (ERC) under the European Union's Horizon 2020 research and innovation programme (SCoTMOF), ERC-2015-StG 677289.

510 Sandia National Laboratories is a multimission laboratory managed and operated by National 511 Technology and Engineering Solutions of Sandia, LLC., a wholly owned subsidiary of Honeywell 512 International, Inc., for the U.S. Department of Energy's National Nuclear Security Administration 513 under contract DE-NA-0003525. The authors gratefully acknowledge funding from the U.S. 514 Department of Energy, Office of Energy Efficiency and Renewable Energy, Hydrogen and Fuel Cell 515 Technologies Office, through the Hydrogen Storage Materials Advanced Research Consortium 516 (HyMARC). This paper describes objective technical results and analysis. Any subjective views or 517 opinions that might be expressed in the paper do not necessarily represent the views of the U.S. 518 Department of Energy or the United States Government.

519 J.D.E acknowledges the support of the Alexander von Humboldt foundation and the Center for 520 Information Services and High Performance Computing (ZIH) at TU Dresden.

521 S.K.G. and S.M. acknowledge SERB (Project No. CRG/2019/000906), India for financial support.

522 K.K. and R.K. acknowledge Active Co. Research Grant for funding.

523 S.K. acknowledge funding from the European Research Council (ERC) under the European Union's 524 Horizon 2020 research and innovation programme (COSMOS), ERC-2017-StG 756489.

525 N.L. and J.G.M acknowledge funding from the European Commission through the H2020-MSCA526 RISE-2019 program (ZEOBIOCHEM - 872102) and the Spanish MICINN and AEI/FEDER 527 (RTI2018-099504-B-C21). N.L. thanks the University of Alicante for funding (UATALENTO17-05).

528 ICN2 is supported by the Severo Ochoa program from the Spanish MINECO (Grant No. SEV-2017529 0706)

530 S.M.J.R. and A.L wish to thank the Fund for Scientific Research Flanders (FWO), under grant nos. 531 12T3519N and 11D2220N.

L.S. was supported by the EPSRC Cambridge NanoDTC EP/L015978/1

C.T.Y. and T.S.N. acknowledges funds from the National Research Foundation of Korea, NRF2017M3A7B4042140 and NRF-2017M3A7B4042235

P.F. and H. Y. acknowledge US Department of Energy, Office of Basic Energy Sciences, Materials Sciences and Engineering Division under Award No. DE-SC0010596 (P.F.).

R.O would like to acknowledge funding support during his Ph.D study from Indonesian Endowment Fund for Education-LPDP with the contract No. 202002220216006 